\title{
q-Szász-Mirakyan-Kantorovich Operators of Functions of Two Variables in Polynomial Weighted Spaces
}

\author{
Mediha Örkcü \\ Department of Mathematics, Faculty of Sciences, Gazi University, Teknikokullar, 06500 Ankara, Turkey \\ Correspondence should be addressed to Mediha Örkcü; medihaakcay@gazi.edu.tr
}

Received 20 June 2013; Accepted 10 November 2013

Academic Editor: Sergei V. Pereverzyev

Copyright @ 2013 Mediha Örkcü. This is an open access article distributed under the Creative Commons Attribution License, which permits unrestricted use, distribution, and reproduction in any medium, provided the original work is properly cited.

The present paper deals with approximation properties of $q$-Szász-Mirakyan-Kantorovich operators. We construct new bivariate generalization by $q_{R}$-integral and these operators' approximation properties in polynomial weighted spaces are investigated. Also, we obtain Voronovskaya-type theorem for the proposed operators in polynomial weighted spaces of functions of two variables.

\section{Introduction}

In the past two decades, $q$-calculus has gained popularity in the construction of linear approximation processes. Lupaş [1] and Phillips [2] defined generalizations of the Bernstein operators called $q$-Bernstein operators. Then, as Phillips has done for Bernstein operators, the authors introduced modifications of the other important operators based on the $q$-integers, for example, $q$-Meyer-König operators $[3,4]$, $q$-Bleimann, Butzer, and Hahn operators [5, 6], q-SzászMirakyan operators [7-9], $q$-Baskakov operators [10, 11].

On the other hand, Stancu [12] first introduced new linear positive operators in two- and several dimensional variables. Recently, Barbosu [13] introduced a Stancu-type generalization of two-dimensional Bernstein operators based on $q$ integers and called them bivariate $q$-Bernstein operators. Doğru and Gupta [14] constructed a bivariate generalization of the Meyer-König and Zeller operators based on the qintegers. Agratini [15] presented two-dimensional extension of some univariate positive approximation processes expressed by series.

All the above mentioned new operators motivate us for current work. In this paper, we firstly extend the q-SzászMirakyan-Kantorovich operators to the case of bivariate functions. Then these operators' approximation properties in polynomial weighted spaces are investigated. Also we obtain Voronovskaya-type theorem for the proposed operators in polynomial weighted spaces of functions of two variables.
Now we recall some definitions about $q$-integers. For any nonnegative integer $r$, the $q$-integer of the number $r$ is defined by

$$
[r]_{q}=\left\{\begin{array}{ll}
1+q+\cdots+q^{r-1} & \text { if } q \neq 1 \\
r & \text { if } q=1,
\end{array} \quad[0]_{q}=1,\right.
$$

where $q$ is a positive real number. The $q$-factorial is defined as

$$
[r]_{q} !=\left\{\begin{array}{ll}
{[1]_{q}[2]_{q} \cdots[r]_{q}} & \text { if } r=1,2, \ldots \\
1 & \text { if } r=0,
\end{array} \quad[0]_{q} !=1 .\right.
$$

Two $q$-analogues of the exponential function $e^{x}$ are given as

$$
\begin{aligned}
& E_{q}(x)=\sum_{n=0}^{\infty} q^{n(n-1) / 2} \frac{x^{n}}{[n]_{q} !}, \quad x \in \mathbb{R}, \\
& \varepsilon_{q}(x)=\sum_{n=0}^{\infty} \frac{x^{n}}{[n]_{q} !}, \quad|x|<\frac{1}{1-q} .
\end{aligned}
$$

The following relation between $q$-exponential functions $E_{q}(x)$ and $\varepsilon_{q}(x)$ holds:

$$
E_{q}(x) \varepsilon_{q}(-x)=1, \quad|x|<\frac{1}{1-q} .
$$


The $q$-derivative of a function $f(x)$, denoted by $D_{q} f$, is defined by

$$
\begin{gathered}
\left(D_{q} f\right)(x)=\frac{f(q x)-f(x)}{(q-1) x}, \quad x \neq 0, \\
\left(D_{q} f\right)(0)=\lim _{x \rightarrow 0}\left(D_{q} f\right)(x) .
\end{gathered}
$$

Also, it is known that $D_{q} E(a x)=a E(q a x)$.

The $q$-integral of the function $f$ over the interval $[0, b]$ is defined by

$$
\int_{0}^{b} f(t) d_{q} t=b(1-q) \sum_{j=0}^{\infty} f\left(b q^{j}\right) q^{j}, \quad 0<q<1 .
$$

If $f$ is integrable over $[0, b]$, then

$$
\lim _{q \rightarrow 1^{-}} \int_{0}^{b} f(t) d_{q} t=\int_{0}^{b} f(t) d t
$$

Generally accepted definition for $q$-integral over an interval $[a, b]$ is

$$
\int_{a}^{b} f(t) d_{q} t=\int_{0}^{b} f(t) d_{q} t-\int_{0}^{a} f(t) d_{q} t .
$$

In order to generalize and spread the existing inequalities, Marinkovic et al. considered new type of the $q$-integral. So, the problems which ensue from the general definition of $q$ integral were overcome. The Riemann-type $q$-integral [16] in the interval $[a, b]$ was defined as

$$
\begin{array}{r}
\int_{a}^{b} f(t) d_{q}^{R} t=(1-q)(b-a) \sum_{j=0}^{\infty} f\left(a+(b-a) q^{j}\right) q^{j} \\
0<q<1 .
\end{array}
$$

This definition includes only point inside the interval of the integration.

Details of $q$-integers can be found in [17].

\section{Construction of the Bivariate Operators}

For $q_{1}, q_{2} \in(0,1)$ and $(m, n) \in \mathbb{N} \times \mathbb{N}$, we now define new operators that we call the $q$-Szász-Mirakyan-Kantorovich operators of functions of two variables as follows:

$$
\begin{aligned}
S_{m, n}^{q_{1}, q_{2}} & (f ; x, y) \\
= & \sum_{l=0}^{\infty} \sum_{k=0}^{\infty} \frac{[m]_{q_{1}}^{k+1} x^{k}}{[k]_{q_{1}} !} \frac{[n]_{q_{2}}^{l+1} y^{l}}{[l]_{q_{2}} !} q_{1}^{k(k-1)-1} q_{2}^{l(l-1)-1} \\
& \times E_{q_{1}}\left(-[m]_{q_{1}} q_{1}^{k} x\right) E_{q_{2}}\left(-[n]_{q_{2}} q_{2}^{l} y\right) \\
& \times \int_{[l]_{q_{2}} / q_{2}^{k-1}[n]_{q_{2}}}^{[l+1]_{q_{2}} / q_{2}^{k-1}[n]_{q_{2}}} \int_{[k]_{q_{1}} / q_{1}^{k-1}[m]_{q_{1}}}^{[k+1]_{q_{1}} / q_{1}^{k-1}[m]_{q_{1}}} f(t, s) d_{q_{1}}^{R} t d_{q_{2}}^{R} s,
\end{aligned}
$$

where

$$
\begin{aligned}
\int_{c}^{d} \int_{a}^{b} f(t, s) d_{q_{1}}^{R} t d_{q_{2}}^{R} s \\
=\left(1-q_{1}\right)\left(1-q_{2}\right)(b-a)(c-d) \\
\quad \times \sum_{j=0}^{\infty} \sum_{i=0}^{\infty} f\left(a+(b-a) q_{1}^{i}, c+(c-d) q_{2}^{j}\right) q_{1}^{i} q_{2}^{j},
\end{aligned}
$$

and $f$ is a $q_{R}$-integrable function, so the series in (11) converges. It is clear that the operators given in (10) are linear and positive. For the operator $S_{m, n}^{q_{1}, q_{2}}$, if $f$ is a $q_{R}$-integrable function and $f(x, y)=f_{1}(x) f_{2}(y),(x, y) \in \mathbb{R}_{+}^{2}$, then

$$
S_{m, n}^{q_{1}, q_{2}}(f(t, s) ; x, y)=S_{m}^{q_{1}}\left(f_{1}(t), x\right) S_{n}^{q_{2}}\left(f_{2}(s), y\right) .
$$

Now, in order to obtain approximation properties of proposed operators, we give some auxiliary results. For a fixed $x \in \mathbb{R}_{+}$, by the $q$-Taylor theorem [18], we write

$$
g(t)=\sum_{k=0}^{\infty} \frac{(t-x)_{q}^{k}}{[k]_{q} !} D_{q}^{k} g(x),
$$

where

$$
(t-x)_{q}^{k}=\prod_{s=0}^{k-1}\left(t-q^{s} x\right)=\sum_{s=0}^{k}\left[\begin{array}{l}
k \\
s
\end{array}\right]_{q} q^{s(s-1) / 2} t^{k-s}(-x)^{s} .
$$

Choosing $t=0$ and taking into account

$$
\begin{gathered}
(-x)_{q}^{k}=(-1)^{k} x^{k} q^{k(k-1) / 2}, \\
D_{q}^{k} E_{q}\left(-[n]_{q} x\right)=\left(-[n]_{q}\right)^{k} q^{k(k-1) / 2} E_{q}\left(-[n]_{q} q^{k} x\right),
\end{gathered}
$$

we get for $g(x)=E_{q}\left(-[n]_{q} x\right)$ that

$$
\begin{aligned}
1 & =g(0)=\sum_{k=0}^{\infty} \frac{(-x)_{q}^{k}}{[k]_{q} !} D_{q}^{k} g(x) \\
& =\sum_{k=0}^{\infty} \frac{\left([n]_{q} x\right)^{k}}{[k]_{q} !} q^{k(k-1)} E_{q}\left(-[n]_{q} q^{k} x\right) .
\end{aligned}
$$

Similarly, choosing $t=0$ and taking into account

$$
\begin{gathered}
(-x)_{q}^{k}=(-1)^{k} x^{k} q^{k(k-1) / 2}, \\
D_{q}^{k} E_{q}\left(-[n]_{q} q x\right)=\left(-[n]_{q} q\right)^{k} q^{k(k-1) / 2} E_{q}\left(-[n]_{q} q^{k+1} x\right),
\end{gathered}
$$

we obtain for $g(x)=E_{q}\left(-[n]_{q} q x\right)$ that

$$
\begin{aligned}
1 & =g(0)=\sum_{k=0}^{\infty} \frac{(-x)_{q}^{k}}{[k]_{q} !} D_{q}^{k} g(x) \\
& =\sum_{k=0}^{\infty} \frac{\left([n]_{q} x\right)^{k}}{[k]_{q} !} q^{k^{2}} E_{q}\left(-[n]_{q} q^{k+1} x\right) .
\end{aligned}
$$


Also, using

$$
\begin{gathered}
(-x)_{q}^{k}=(-1)^{k} x^{k} q^{k(k-1) / 2} \\
D_{q}^{k} E_{q}\left(-[n]_{q} q^{2} x\right)=\left(-[n]_{q}\right)^{k} q^{2 k} q^{k(k-1) / 2} E_{q}\left(-[n]_{q} q^{k+2} x\right),
\end{gathered}
$$

we have for $g(x)=E_{q}\left(-[n]_{q} q^{2} x\right)$ that

$$
\begin{aligned}
1 & =g(0)=x \sum_{k=0}^{\infty} \frac{(-x)_{q}^{k}}{[k]_{q} !} D_{q}^{k} g(x) \\
& =\sum_{k=0}^{\infty} \frac{\left([n]_{q} x\right)^{k}}{[k]_{q} !} q^{k^{2}} E_{q}\left(-[n]_{q} q^{k+2} x\right) .
\end{aligned}
$$

Lemma 1. Let $q_{1}, q_{2} \in(0,1)$ and $(m, n) \in \mathbb{N} \times \mathbb{N}$. One has

$$
\begin{gathered}
S_{m, n}^{q_{1}, q_{2}}(1 ; x, y)=1 \\
S_{m, n}^{q_{1}, q_{2}}(t ; x)=x+\frac{q_{1}}{[2]_{q_{1}}[m]_{q_{1}}}, \\
S_{m, n}^{q_{1}, q_{2}}(s ; y)=y+\frac{q_{2}}{[2]_{q_{2}}[n]_{q_{2}}}, \\
S_{m, n}^{q_{1}, q_{2}}\left(t^{2} ; x\right)=x^{2}+\frac{1}{[m]_{q_{1}}}\left(1+\frac{2 q_{1}}{[2]_{q_{1}}}\right) x+\frac{q_{1}^{2}}{[3]_{q_{1}}[m]_{q_{1}}^{2}}, \\
S_{m, n}^{q_{1}, q_{2}}\left(s^{2} ; y\right)=y^{2}+\frac{1}{[n]_{q_{2}}}\left(1+\frac{2 q_{2}}{[2]_{q_{2}}}\right) y+\frac{q_{2}^{2}}{[3]_{q_{2}}[n]_{q_{2}}^{2}} .
\end{gathered}
$$

Proof. Using $[k+1]_{q}=[k]_{q}+q^{k}$ and from $\int_{[l]_{q_{2}} / q_{2}^{k-1}[n]_{q_{2}}}^{[l+1]_{q_{2}} / q_{2}^{k-1}[n]_{q_{2}}} \int_{[k]_{q_{1}} / q_{1}^{k-1}[m]_{q_{1}}}^{[k+1]_{q_{1}} / q_{1}^{k-1}[m]_{q_{1}}} d_{q_{1}}^{R} t d_{q_{2}}^{R} s=q_{1} q_{2} /[m]_{q_{1}}[n]_{q_{2}}$, we can write

$$
\begin{aligned}
S_{m, n}^{q_{1}, q_{2}}(1 ; x, y)= & \sum_{l=0}^{\infty} \sum_{k=0}^{\infty} \frac{[m]_{q_{1}}^{k} x^{k}}{[k]_{q_{1}} !} \frac{[n]_{q_{2}}^{l} y^{l}}{[l]_{q_{2}} !} q_{1}^{k(k-1)} q_{2}^{l(l-1)} \\
& \times E_{q_{1}}\left(-[m]_{q_{1}} q_{1}^{k} x\right) E_{q_{2}}\left(-[n]_{q_{2}} q_{2}^{l} y\right) .
\end{aligned}
$$

$S_{m, n}^{q_{1}, q_{2}}(1 ; x, y)=1$ is obtained from the above identity (16).

Now, taking $f(t, s)=t$ and since $\int_{[l]_{q_{2}} / q_{2}^{k-1}[n]_{q_{2}}}^{[l+1]_{q_{2}} / q_{q_{2}}^{k-1}[n]_{q_{2}}} \int_{[k]_{q_{1}} / q_{1}^{k-1}[m]_{q_{1}}}^{[k+1]_{q_{1}} / q_{1}^{k-1}[m]_{q_{1}}} t d_{q_{1}}^{R} t d_{q_{2}}^{R} s=\left(q_{1} q_{2} /[m]_{q_{1}}[n]_{q_{2}}\right)$ $\left(\left([k]_{q_{1}} / q_{1}^{k-1}[m]_{q_{1}}\right)+\left(q_{1} /[2]_{q_{1}}[m]_{q_{1}}\right)\right)$, we get from the linearity of $S_{m, n}^{q_{1}, q_{2}}$ that

$$
\begin{aligned}
S_{m, n}^{q_{1}, q_{2}}(t ; x)= & \sum_{l=0}^{\infty} \sum_{k=0}^{\infty} \frac{[m]_{q_{1}}^{k} x^{k}}{[k]_{q_{1}} !} \frac{[n]_{q_{2}}^{l} y^{l}}{[l]_{q_{2}} !} q_{1}^{k(k-1)} q_{2}^{l(l-1)} \\
& \times E_{q_{1}}\left(-[m]_{q_{1}} q_{1}^{k} x\right) E_{q_{2}}\left(-[n]_{q_{2}} q_{2}^{l} y\right) \frac{[k]_{q_{1}}}{q_{1}^{k-1}[m]_{q_{1}}} \\
& +\sum_{l=0}^{\infty} \sum_{k=0}^{\infty} \frac{[m]_{q_{1}}^{k} x^{k}}{[k]_{q_{1}} !} \frac{[n]_{q_{2}}^{l} y^{l}}{[l]_{q_{2}} !} q_{1}^{k(k-1)} q_{2}^{l(l-1)} \\
& \times E_{q_{1}}\left(-[m]_{q_{1}} q_{1}^{k} x\right) E_{q_{2}}\left(-[n]_{q_{2}} q_{2}^{l} y\right) \frac{q_{1}}{[2]_{q_{1}}[m]_{q_{1}}}
\end{aligned}
$$

From this, applying (16) and (18), we have

$$
\begin{aligned}
S_{m, n}^{q_{1}, q_{2}}( & t ; x) \\
= & \sum_{l=0}^{\infty} \sum_{k=1}^{\infty} \frac{[m]_{q_{1}}^{k-1} x^{k}}{[k-1]_{q_{1}} !} \frac{[n]_{q_{2}}^{l} y^{l}}{[l]_{q_{2}} !} \frac{q_{1}^{k(k-1)}}{q_{1}^{k-1}} q_{2}^{l(l-1)} \\
& \times E_{q_{1}}\left(-[m]_{q_{1}} q_{1}^{k} x\right) E_{q_{2}}\left(-[n]_{q_{2}} q_{2}^{l} y\right)+\frac{q_{1}}{[2]_{q_{1}}[m]_{q_{1}}} \\
= & x \sum_{l=0}^{\infty} \sum_{k=0}^{\infty} \frac{[m]_{q_{1}}^{k} x^{k}}{[k]_{q_{1}} !} \frac{[n]_{q_{2}}^{l} y^{l}}{[l]_{q_{2}} !} q_{1}^{k^{2}} q_{2}^{l(l-1)} \\
& \times E_{q_{1}}\left(-[m]_{q_{1}} q_{1}^{k+1} x\right) E_{q_{2}}\left(-[n]_{q_{2}} q_{2}^{l} y\right)+\frac{q_{1}}{[2]_{q_{1}}[m]_{q_{1}}} \\
= & x+\frac{q_{1}}{[2]_{q_{1}}[m]_{q_{1}}} .
\end{aligned}
$$

Similarly, we write that

$$
S_{m, n}^{q_{1}, q_{2}}(s ; y)=y+\frac{q_{2}}{[2]_{q_{2}}[n]_{q_{2}}} .
$$

Now, taking $f(t, s)=t^{2}$ and from

$$
\begin{aligned}
\int_{[l]_{q_{2}} / q_{2}^{k-1}[n]_{q_{2}}}^{[l+1]_{q_{2}} / q_{2}^{k-1}[n]_{q_{2}}} \int_{[k]_{q_{1}} / q_{1}^{k-1}[m]_{q_{1}}}^{[k+1]_{q_{1}} / q_{1}^{k-1}[m]_{q_{1}}} t^{2} d_{q_{1}}^{R} t d_{q_{2}}^{R} s \\
=\frac{q_{1} q_{2}}{[m]_{q_{1}}[n]_{q_{2}}} \\
\quad \times\left(\frac{[k]_{q_{1}}^{2}}{q_{1}^{2 k-2}[m]_{q_{1}}^{2}}+\frac{[k]_{q_{1}}}{q_{1}^{k-1}[m]_{q_{1}}} \frac{2 q_{1}}{[2]_{q_{1}}[m]_{q_{1}}}+\frac{q_{1}^{2}}{[3]_{q_{1}}[m]_{q_{1}}^{2}}\right),
\end{aligned}
$$


we have

$$
\begin{aligned}
& S_{m, n}^{q_{1}, q_{2}}\left(t^{2} ; x\right) \\
& =\sum_{l=0}^{\infty} \sum_{k=1}^{\infty} \frac{[m]_{q_{1}}^{k-1} x^{k}}{[k-1]_{q_{1}} !} \frac{[n]_{q_{2}}^{l} y^{l}}{[l]_{q_{2}} !} q_{1}^{k(k-1)} q_{2}^{l(l-1)} \\
& \quad \times E_{q_{1}}\left(-[m]_{q_{1}} q_{1}^{k} x\right) E_{q_{2}}\left(-[n]_{q_{2}} q_{2}^{l} y\right) \frac{[k]_{q_{1}}}{q_{1}^{2 k-2}[m]_{q_{1}}} \\
& \quad+\frac{2 q_{1}}{[2]_{q_{1}}[m]_{q_{1}}} x+\frac{q_{1}^{2}}{[3]_{q_{1}}[m]_{q_{1}}^{2}}
\end{aligned}
$$

From this, using $[k]_{q}=[k-1]_{q}+q^{k-1}$ we get

$$
\begin{aligned}
S_{m, n}^{q_{1}, q_{2}}\left(t^{2} ; x\right)= & \sum_{l=0}^{\infty} \sum_{k=2}^{\infty} \frac{[m]_{q_{1}}^{k-2} x^{k}}{[k-2]_{q_{1}} !} \frac{[n]_{q_{2}}^{l} y^{l}}{[l]_{q_{2}} !} q_{1}^{k(k-1)} q_{2}^{l(l-1)} \\
& \times E_{q_{1}}\left(-[m]_{q_{1}} q_{1}^{k} x\right) E_{q_{2}}\left(-[n]_{q_{2}} q_{2}^{l} y\right) \frac{1}{q_{1}^{2 k-2}} \\
& +\sum_{l=0}^{\infty} \sum_{k=1}^{\infty} \frac{[m]_{q_{1}}^{k-1} x^{k}}{[k-1]_{q_{1}} !} \frac{[n]_{q_{2}}^{l} y^{l}}{[l]_{q_{2}} !} q_{1}^{k(k-1)} q_{2}^{l(l-1)} \\
& \times E_{q_{1}}\left(-[m]_{q_{1}} q_{1}^{k} x\right) E_{q_{2}}\left(-[n]_{q_{2}} q_{2}^{l} y\right) \frac{1}{[m]_{q_{1}} q_{1}^{k-1}} \\
& +\frac{2 q_{1}}{[2]_{q_{1}}[m]_{q_{1}}} x+\frac{q_{1}^{2}}{[3]_{q_{1}}[m]_{q_{1}}^{2}} .
\end{aligned}
$$

Replacing $k$ by $k+2$ and $k$ by $k+1$ in the abovementioned, we obtain

$$
\begin{aligned}
S_{m, n}^{q_{1}, q_{2}}\left(t^{2} ; x\right)= & x^{2} \sum_{l=0}^{\infty} \sum_{k=0}^{\infty} \frac{[m]_{q_{1}}^{k} x^{k}}{[k]_{q_{1}} !} \frac{[n]_{q_{2}}^{l} y^{l}}{[l]_{q_{2}} !} q_{1}^{(k+2)(k+1)} q_{2}^{l(l-1)} \\
& \times E_{q_{1}}\left(-[m]_{q_{1}} q_{1}^{k+2} x\right) E_{q_{2}}\left(-[n]_{q_{2}} q_{2}^{l} y\right) \frac{1}{q_{1}^{2 k+2}} \\
& +x \sum_{l=0}^{\infty} \sum_{k=0}^{\infty} \frac{[m]_{q_{1}}^{k} x^{k}}{[k]_{q_{1}} !} \frac{[n]_{q_{2}}^{l} y^{l}}{[l]_{q_{2}} !} q_{1}^{k^{2}} q_{2}^{l(l-1)} \\
& \times E_{q_{1}}\left(-[m]_{q_{1}} q_{1}^{k+1} x\right) E_{q_{2}}\left(-[n]_{q_{2}} q_{2}^{l} y\right) \frac{1}{[m]_{q_{1}}} \\
& +\frac{2 q_{1}}{[2]_{q_{1}}[m]_{q_{1}}} x+\frac{q_{1}^{2}}{[3]_{q_{1}}[m]_{q_{1}}^{2}} .
\end{aligned}
$$

Equation (20) implies

$$
S_{m, n}^{q_{1}, q_{2}}\left(t^{2} ; x\right)=x^{2}+\frac{1}{[m]_{q_{1}}}\left(1+\frac{2 q_{1}}{[2]_{q_{1}}}\right) x+\frac{q_{1}^{2}}{[3]_{q_{1}}[m]_{q_{1}}^{2}} .
$$

Similarly, we write that

$$
S_{m, n}^{q_{1}, q_{2}}\left(s^{2} ; y\right)=y^{2}+\frac{1}{[n]_{q_{2}}}\left(1+\frac{2 q_{2}}{[2]_{q_{2}}}\right) y+\frac{q_{2}^{2}}{[3]_{q_{2}}[n]_{q_{2}}^{2}} .
$$

So, the proof is completed.

Similarly, given by the proof of Lemma 1, we calculate $S_{m, n}^{q_{1}, q_{2}}\left(t^{3} ; x\right)$ and $S_{m, n}^{q_{1}, q_{2}}\left(t^{4} ; x\right)$, shortly. Since

$$
\begin{aligned}
\int_{[l]_{q_{2}} / q_{2}^{k-1}[n]_{q_{2}}}^{[l+1]_{q_{2}} / q_{2}^{k-1}[n]_{q_{2}}} \int_{[k]_{q_{1}} / q_{1}^{k-1}[m]_{q_{1}}}^{[k+1]_{q_{1}} / q_{1}^{k-1}[m]_{q_{1}}} t^{3} d_{q_{1}}^{R} t d_{q_{2}}^{R} s \\
=\frac{q_{1} q_{2}}{[m]_{q_{1}}[n]_{q_{2}}}\left(\frac{[k]_{q_{1}}^{3}}{q_{1}^{3 k-3}[m]_{q_{1}}^{3}}+\frac{3[k]_{q_{1}}^{2}}{q_{1}^{2 k-2}[m]_{q_{1}}^{2}} \frac{q_{1}}{[2]_{q_{1}}[m]_{q_{1}}}\right. \\
\left.+\frac{3[k]_{q_{1}}}{q_{1}^{k-1}[m]_{q_{1}}} \frac{q_{1}^{2}}{[3]_{q_{1}}[m]_{q_{1}}^{2}}+\frac{q_{1}^{3}}{[4]_{q_{1}}[m]_{q_{1}}^{3}}\right)
\end{aligned}
$$

we write

$$
\begin{aligned}
S_{m, n}^{q_{1}, q_{2}}( & \left.t^{3} ; x\right) \\
= & \sum_{l=0}^{\infty} \sum_{k=1}^{\infty} \frac{[m]_{q_{1}}^{k-1} x^{k}}{[k-1]_{q_{1}} !} \frac{[n]_{q_{2}}^{l} y^{l}}{[l]_{q_{2}} !} q_{1}^{k(k-1)} q_{2}^{l(l-1)} \\
& \times E_{q_{1}}\left(-[m]_{q_{1}} q_{1}^{k} x\right) E_{q_{2}}\left(-[n]_{q_{2}} q_{2}^{l} y\right) \\
& \times \frac{[k-1]_{q_{1}}^{2}+2 q^{k-1}[k-1]+q^{2 k-2}}{q_{1}^{3 k-3}[m]_{q_{1}}^{2}} \\
& +\frac{3 q_{1}}{[2]_{q_{1}}[m]_{q_{1}}}\left(x^{2}+\frac{1}{[m]_{q_{1}}} x\right) \\
& +\frac{3 q_{1}^{2}}{[3]_{q_{1}}[m]_{q_{1}}^{2}} x+\frac{q_{1}^{3}}{[4]_{q_{1}}[m]_{q_{1}}^{3}} .
\end{aligned}
$$

Using $[k]_{q}=[k-1]_{q}+q^{k-1}$, we have

$$
\begin{aligned}
\sum_{l=0}^{\infty} \sum_{k=1}^{\infty} \frac{[m]_{q_{1}}^{k-1} x^{k}}{[k-1]_{q_{1}} !} \frac{[n]_{q_{2}}^{l} y^{l}}{[l]_{q_{2}} !} q_{1}^{k(k-1)} q_{2}^{l(l-1)} \\
\quad \times E_{q_{1}}\left(-[m]_{q_{1}} q_{1}^{k} x\right) E_{q_{2}}\left(-[n]_{q_{2}} q_{2}^{l} y\right)
\end{aligned}
$$




$$
\begin{aligned}
& \times \frac{[k-1]_{q_{1}}^{2}+2 q^{k-1}[k-1]+q^{2 k-2}}{q_{1}^{3 k-3}[m]_{q_{1}}^{2}} \\
& =\sum_{l=0}^{\infty} \sum_{k=3}^{\infty} \frac{[m]_{q_{1}}^{k-3} x^{k}}{[k-3]_{q_{1}} !} \frac{[n]_{q_{2}}^{l} y^{l}}{[l]_{q_{2}} !} q_{1}^{k(k-1)} q_{2}^{l(l-1)} \\
& \times E_{q_{1}}\left(-[m]_{q_{1}} q_{1}^{k} x\right) E_{q_{2}}\left(-[n]_{q_{2}} q_{2}^{l} y\right) \frac{1}{q_{1}^{3 k-3}} \\
& +\sum_{l=0}^{\infty} \sum_{k=2}^{\infty} \frac{[m]_{q_{1}}^{k-2} x^{k}}{[k-2]_{q_{1}} !} \frac{[n]_{q_{2}}^{l} y^{l}}{[l]_{q_{2}} !} q_{1}^{k(k-1)} q_{2}^{l(l-1)} \\
& S_{m, n}^{q_{1}, q_{2}}\left(t^{3} ; x\right)=x^{3}+\frac{1}{[m]_{q_{1}}}\left(\frac{1}{q_{1}}+2\right) x^{2}+\frac{1}{[m]_{q_{1}}^{2}} x \\
& +\frac{3 q_{1}}{[2]_{q_{1}}[m]_{q_{1}}}\left(x^{2}+\frac{1}{[m]_{q_{1}}} x\right) \\
& +\frac{3 q_{1}^{2}}{[3]_{q_{1}}[m]_{q_{1}}^{2}} x+\frac{q_{1}^{3}}{[4]_{q_{1}}[m]_{q_{1}}^{3}} \text {. }
\end{aligned}
$$$$
\times E_{q_{1}}\left(-[m]_{q_{1}} q_{1}^{k} x\right) E_{q_{2}}\left(-[n]_{q_{2}} q_{2}^{l} y\right) \frac{q_{1}^{k-2}}{q_{1}^{3 k-3}[m]_{q_{1}}}
$$$$
+\sum_{l=0}^{\infty} \sum_{k=2}^{\infty} \frac{[m]_{q_{1}}^{k-2} x^{k}}{[k-2]_{q_{1}} !} \frac{[n]_{q_{2}}^{l} y^{l}}{[l]_{q_{2}} !} q_{1}^{k(k-1)} q_{2}^{l(l-1)}
$$$$
\times E_{q_{1}}\left(-[m]_{q_{1}} q_{1}^{k} x\right) E_{q_{2}}\left(-[n]_{q_{2}} q_{2}^{l} y\right) \frac{2 q^{k-1}}{q_{1}^{3 k-3}[m]_{q_{1}}}
$$$$
+\sum_{l=0}^{\infty} \sum_{k=1}^{\infty} \frac{[m]_{q_{1}}^{k-1} x^{k}}{[k-1]_{q_{1}} !} \frac{[n]_{q_{2}}^{l} y^{l}}{[l]_{q_{2}} !} q_{1}^{k(k-1)} q_{2}^{l(l-1)}
$$$$
\times E_{q_{1}}\left(-[m]_{q_{1}} q_{1}^{k} x\right) E_{q_{2}}\left(-[n]_{q_{2}} q_{2}^{l} y\right) \frac{q^{2 k-2}}{q_{1}^{3 k-3}[m]_{q_{1}}^{2}} .
$$

Then, we rewrite

$$
\begin{aligned}
= & x^{3} \sum_{l=0}^{\infty} \sum_{k=0}^{\infty} \frac{[m]_{q_{1}}^{k} x^{k}}{[k]_{q_{1}} !} \frac{[n]_{q_{2}}^{l} y^{l}}{[l]_{q_{2}} !} q_{1}^{(k+3)(k+2)} q_{2}^{l(l-1)} \\
& \times E_{q_{1}}\left(-[m]_{q_{1}} q_{1}^{k+3} x\right) E_{q_{2}}\left(-[n]_{q_{2}} q_{2}^{l} y\right) \frac{1}{q_{1}^{3 k+6}} \\
& +x^{2} \sum_{l=0 k=0}^{\infty} \sum^{\infty} \frac{[m]_{q_{1}}^{k} x^{k}}{[k]_{q_{1}} !} \frac{[n]_{q_{2}}^{l} y^{l}}{[l]_{q_{2}} !} q_{1}^{(k+2)(k+1)} q_{2}^{l(l-1)} \\
& \times E_{q_{1}}\left(-[m]_{q_{1}} q_{1}^{k+2} x\right) E_{q_{2}}\left(-[n]_{q_{2}} q_{2}^{l} y\right) \frac{q_{1}^{k}}{q_{1}^{3 k+3}[m]_{q_{1}}} \\
& +x^{2} \sum_{l=0 k=0}^{\infty} \sum^{\infty} \frac{[m]_{q_{1}}^{k} x^{k}}{[k]_{q_{1}} !} \frac{[n]_{q_{2}}^{l} y^{l}}{[l]_{q_{2}} !} q_{1}^{(k+2)(k+1)} q_{2}^{l(l-1)} \\
& \times E_{q_{1}}\left(-[m]_{q_{1}} q_{1}^{k+2} x\right) E_{q_{2}}\left(-[n]_{q_{2}} q_{2}^{l} y\right) \frac{2 q^{k+1}}{q_{1}^{3 k+3}[m]_{q_{1}}} \\
& +x \sum_{l=0}^{\infty} \sum_{k=0}^{\infty} \frac{[m]_{q_{1}}^{k} x^{k}}{[k]_{q_{1}} !} \frac{[n]_{q_{2}}^{l} y^{l}}{[l]_{q_{2}} !} q_{1}^{(k+1)(k)} q_{2}^{l(l-1)} \\
& \left(-[m]_{q_{1}} q_{1}^{k+1} x\right) E_{q_{2}}\left(-[n]_{q_{2}} q_{2}^{l} y\right) \frac{q^{2 k}}{q_{1}^{3 k}[m]_{q_{1}}^{2}} .
\end{aligned}
$$

Since

$$
\begin{aligned}
\int_{[l]_{q_{2}} / q_{2}^{k-1}[n]_{q_{2}}}^{[l+1]_{q_{2}} / q_{2}^{k-1}[n]_{q_{2}}} \int_{[k]_{q_{1}} / q_{1}^{k-1}[m]_{q_{1}}}^{[k+1]_{q_{1}} / q_{1}^{k-1}[m]_{q_{1}}} t^{4} d_{q_{1}}^{R} t d_{q_{2}}^{R} s \\
=\frac{q_{1} q_{2}}{[m]_{q_{1}}[n]_{q_{2}}}\left(\frac{[k]_{q_{1}}^{4}}{q_{1}^{4 k-4}[m]_{q_{1}}^{4}}+\frac{4[k]_{q_{1}}^{3}}{q_{1}^{3 k-3}[m]_{q_{1}}^{3}} \frac{q_{1}}{[2]_{q_{1}}[m]_{q_{1}}}\right. \\
+\frac{6[k]_{q_{1}}^{2}}{q_{1}^{2 k-2}[m]_{q_{1}}^{2}} \frac{q_{1}^{2}}{[3]_{q_{1}}[m]_{q_{1}}^{2}} \\
\left.+\frac{4[k]_{q_{1}}}{q_{1}^{k-1}[m]_{q_{1}}} \frac{q_{1}^{3}}{[4]_{q_{1}}[m]_{q_{1}}^{3}}+\frac{q_{1}^{4}}{[5]_{q_{1}}[m]_{q_{1}}^{4}}\right)
\end{aligned}
$$

we obtain

$S_{m, n}^{q_{1}, q_{2}}\left(t^{4} ; x\right)$

$$
\begin{aligned}
= & x^{4}+\frac{1}{[m]_{q_{1}}}\left(\frac{1}{q_{1}^{2}}+\frac{2}{q_{1}}+3\right) x^{3} \\
& +\frac{1}{[m]_{q_{1}}^{2}}\left(\frac{1}{q_{1}^{2}}+\frac{3}{q_{1}}+3\right) x^{2}+\frac{1}{[m]_{q_{1}}^{3}} x \\
& +\frac{4 q_{1}}{[2]_{q_{1}}[m]_{q_{1}}}\left(x^{3}+\frac{1}{[m]_{q_{1}}}\left(\frac{1}{q_{1}}+2\right) x^{2}+\frac{1}{[m]_{q_{1}}^{2}} x\right) \\
& +\frac{6 q_{1}^{2}}{[3]_{q_{1}}[m]_{q_{1}}^{2}}\left(x^{2}+\frac{1}{[m]_{q_{1}}} x\right) \\
& +\frac{4 q_{1}^{3}}{[4]_{q_{1}}[m]_{q_{1}}^{3}} x+\frac{q_{1}^{4}}{[5]_{q_{1}}[m]_{q_{1}}^{4}} .
\end{aligned}
$$

\section{Approximation Properties in Polynomial Weighted Spaces}

For bivariate operators, the space is considered as follows:

$$
\begin{aligned}
C_{p, q}\left(\mathbb{R}_{+}^{2}\right) \\
=\left\{f \in C\left(\mathbb{R}_{+}^{2}\right): \omega_{p, q} f\right. \text { is uniformly continuous and } \\
\left.\quad \text { bounded on } \mathbb{R}_{+}^{2}=[0, \infty) \times[0, \infty)\right\}
\end{aligned}
$$


associated with the weighted function $\omega_{p, q}(x, y)=$ $\omega_{p}(x) \omega_{q}(y),(p, q) \in \mathbb{N}_{0} \times \mathbb{N}_{0}$. The weight $\omega_{p}$ is defined as $\omega_{0}(t)=1, \omega_{p}(x)=\left(1+x^{p}\right)^{-1}$. The norm of this space is denoted by $\|\cdot\|_{p, q}$ and is defined by

$$
\|f\|_{p, q}=\sup _{(x, y) \in \mathbb{R}_{+}^{2}} \omega_{p, q}(x, y)|f(x, y)| .
$$

Now, we give some useful results given by Agratini [15].

For each $z \in \mathbb{R}_{+}$, define the function $\varphi_{z}$ by $\varphi_{z}^{r}(t)=$ $(t-z)^{r}, t \in \mathbb{R}_{+}, r \in \mathbb{N}$. For the one-dimensional operator $L_{s}, s \in \mathbb{N}$, and for each $r \in \mathbb{N}$, a polynomial $\Gamma_{r}$ exists such that

$$
s^{r / 2}\left(L_{s} \varphi_{z}^{r}\right)(t) \leq \Gamma_{r}(t), \quad \operatorname{deg}\left(\Gamma_{r}\right) \leq r
$$

Theorem 2 (see [15]). Consider $(p, q) \in \mathbb{N}_{0} \times \mathbb{N}_{0}$. For any $(m, n) \in \mathbb{N} \times \mathbb{N}$, given by

$$
\begin{aligned}
& \left(L_{m, n} f\right)(x, y) \\
& \quad=\sum_{i=0}^{\infty} \sum_{j=0}^{\infty} a_{m, i}(x) b_{n, j}(y) f\left(x_{m, i}, y_{n, j}\right), \quad(x, y) \in \mathbb{R}_{+}^{2}
\end{aligned}
$$

the operator $L_{m, n}$ verifies

$$
\begin{gathered}
\left\|L_{m, n}\left(\frac{1}{\omega_{p, q}} ; \cdot\right)\right\|_{p, q} \leq c(p, q), \\
\left\|L_{m, n}(f ; \cdot)\right\|_{p, q} \leq c(p, q)\|f\|_{p, q}, \quad f \in C_{p, q}\left(\mathbb{R}_{+}^{2}\right) .
\end{gathered}
$$

Theorem 3 (see $[15]$ ). Let $(p, q) \in \mathbb{N}_{0} \times \mathbb{N}_{0}$. For any $(m, n) \in$ $\mathbb{N} \times \mathbb{N}$, the operator $L_{m, n}$ given by (42) satisfies

$$
\begin{gathered}
\omega_{p, q}(x, y)\left|\left(L_{m, n} f\right)(x, y)-f(x, y)\right| \\
\leq c(p, q) \omega_{f}\left(\frac{\phi(x)}{\sqrt{m}}, \frac{\phi(y)}{\sqrt{n}}\right),
\end{gathered}
$$

$(x, y) \in \mathbb{R}_{+}^{2}$, where $\phi$ is given by

$$
\phi(t)=\sqrt{\Gamma_{2}(t)}+\sqrt{\Gamma_{2}(t)+\sum_{k=0}^{p}\left(\begin{array}{l}
p \\
k
\end{array}\right) \Gamma_{k+2}(t)},
$$

with the polynomials $\Gamma_{\nu}(t), v=\overline{2, p+2}$, being indicated at (41), and $c(p, q)$ is a suitable constant.

Theorem 4 (see $[15])$. Let $(p, q) \in \mathbb{N}_{0} \times \mathbb{N}_{0}$. Let the operator $L_{m, n},(m, n) \in \mathbb{N} \times \mathbb{N}$, be defined by (42). For any $(x, y) \in \mathbb{R}_{+}^{2}$ the pointwise convergence takes place

$$
\lim _{m, n \rightarrow \infty}\left(L_{m, n} f\right)(x, y)=f(x, y), \quad f \in C_{p, q}\left(\mathbb{R}_{+}^{2}\right) .
$$

If $K_{1}, K_{2}$ are compact intervals included in $\mathbb{R}_{+}$, then (46) holds uniformly on the domain $K_{1} \times K_{2}$.

In the latter paper, we use the weight function $\rho(x, y)=$ $\left(1+x^{2}+y^{2}\right)^{-1}$ instead of $\omega_{p, q}(x, y)$ and instead of the space $C_{p, q}\left(\mathbb{R}_{+}^{2}\right)$, the space $C_{2}\left(\mathbb{R}_{+}^{2}\right)$ associated with the weighted function $\rho(x, y)$ is used. We denote the norm of this space by $\|\cdot\|_{2}$.
Lemma 5. The operator $S_{m, n}^{q_{1}, q_{2}},(m, n) \in \mathbb{N} \times \mathbb{N}, q_{1}, q_{2} \in(0,1)$, given by (10) verifies

$$
\begin{gathered}
\left\|S_{m, n}^{q_{1}, q_{2}}\left(\frac{1}{\rho(t, s)} ;\right)\right\|_{2} \leq \frac{10}{3}, \\
\left\|S_{m, n}^{q_{1}, q_{2}}(f ; \cdot)\right\|_{2} \leq \frac{10}{3}\|f\|_{2}, \quad f \in C_{2}\left(\mathbb{R}_{+}^{2}\right) .
\end{gathered}
$$

Proof. Since $q_{1}, q_{2} \in(0,1)$ and by Lemma 1 , we have

$$
\begin{aligned}
& \rho(x, y) S_{m, n}^{q_{1}, q_{2}}\left(\frac{1}{\rho(t, s)} ; \cdot\right) \\
& \quad \leq \rho(x, y)\left(1+x^{2}+y^{2}+\frac{7}{3}(x+y)\right) .
\end{aligned}
$$

So, inequality (47) is proved. Since the operator $S_{m, n}^{q_{1}, q_{2}}$ is linear and positive and by using (47)

$$
\begin{aligned}
\rho(x, y) S_{m, n}^{q_{1}, q_{2}}(f ; \cdot) & \leq \rho(x, y) S_{m, n}^{q_{1}, q_{2}}\left(\rho(t, s)|f| \frac{1}{\rho(t, s)} ; \cdot\right) \\
& \leq \frac{10}{3}\|f\|_{2},
\end{aligned}
$$

we obtain inequality (48).

The Steklov function associated with $f \in C\left(\mathbb{R}_{+}^{2}\right)$ is given as follows:

$$
f_{h, \delta}(x, y)=\frac{1}{h \delta} \int_{0}^{h} d u \int_{0}^{\delta} f(x+u, y+v) d v, \quad(x, y) \in \mathbb{R}_{+}^{2},
$$

where $h, \delta>0$.

The modulus of smoothness function associated with any function $f \in C_{2}\left(\mathbb{R}_{+}^{2}\right)$ is given by

$$
\begin{aligned}
\omega_{f} & (h, \delta) \\
& =\sup _{\substack{0 \leq u \leq h \\
0 \leq v \leq \delta}}\|f(x+u, y+v)-f(x, y)\|_{2}, \quad(x, y) \in \mathbb{R}_{+}^{2} .
\end{aligned}
$$

One can see that

$$
\begin{aligned}
\| f & -f_{h, \delta} \|_{2} \\
= & \sup _{(x, y) \in \mathbb{R}_{+}^{2}} \rho(x, y) \mid \frac{1}{h \delta} \int_{0}^{h} d u \int_{0}^{\delta} f(x+u, y+v) \\
& -f(x, y) d v \mid \\
\leq & \sup _{(x, y) \in \mathbb{R}_{+}^{2}} \rho(x, y) \sup _{0 \leq u \leq h} f(x+u, y+v)-f(x, y) \\
= & \sup _{0 \leq v \leq \delta}\|f(x+u, y+v)-f(x, y)\|_{2} \\
& 0 \leq u \leq h \\
= & \omega_{f}(h, \delta) .
\end{aligned}
$$


The following inequalities verify

$$
\left\|\frac{\partial}{\partial x} f_{h, \delta}\right\|_{2} \leq \frac{2}{h} \omega_{f}(h, \delta), \quad\left\|\frac{\partial}{\partial y} f_{h, \delta}\right\|_{2} \leq \frac{2}{\delta} \omega_{f}(h, \delta),
$$

where $h, \delta>0$. In order to justify these inequalities, one can see that

$$
\begin{aligned}
& \left\|\frac{\partial}{\partial x} f_{h, \delta}\right\|_{2} \\
& =\sup _{(x, y) \in \mathbb{R}_{+}^{2}} \rho(x, y) \frac{1}{h \delta} \mid \int_{0}^{\delta} f(x+h, y+v) \\
& =\sup _{(x, y) \in \mathbb{R}_{+}^{2}} \rho(x, y) \frac{1}{h \delta} \mid \int_{0}^{\delta} f(x+h, y+v)-f(x, y) \\
& \quad+\frac{1}{h \delta} \sup _{(x, y) \in \mathbb{R}_{+}^{2}} \rho(x, y) \int_{0}^{\delta}|f(x+h, y+v)-f(x, y)| d v \mid \\
& \quad+\frac{1}{h \delta} \sup _{(x, y) \in \mathbb{R}_{+}^{2}} \rho(x, y) \int_{0}^{\delta}|f(x, y+v)-f(x, y)| d v \\
& \leq \frac{2}{h} \sup _{(x, y) \in \mathbb{R}_{+}^{2}} \rho(x, y) \sup _{0 \leq u \leq h} f(x+u, y+v)-f(x, y) \\
& 0 \leq v \leq \delta \\
& =\frac{2}{h} \omega_{f}(h, \delta) .
\end{aligned}
$$

Theorem 6. For any $(m, n) \in \mathbb{N} \times \mathbb{N}$ and $f \in C_{2}\left(\mathbb{R}_{+}^{2}\right)$, the operator $S_{m, n}^{q_{1}, q_{2}} q_{1}, q_{2} \in(0,1)$ given by (10) satisfies

$$
\begin{aligned}
& \rho(x, y)\left|\left(S_{m, n}^{q_{1}, q_{2}} f\right)(x, y)-f(x, y)\right| \\
& \leq \frac{10}{3} \omega_{f}\left(\frac{\phi\left(x, q_{1}\right)}{[m]_{q_{1}}}, \frac{\phi\left(y, q_{2}\right)}{[n]_{q_{2}}}\right),
\end{aligned}
$$

where $(x, y) \in \mathbb{R}_{+}^{2}, \phi(x, q)=q /[2]_{q}+\Gamma(x, q)$.

Proof. For any $(m, n) \in \mathbb{N} \times \mathbb{N}$, we can write

$$
\begin{aligned}
\rho(x, y) & \left|\left(S_{m, n}^{q_{1}, q_{2}} f\right)(x, y)-f(x, y)\right| \\
\leq & \rho(x, y)\left|S_{m, n}^{q_{1}, q_{2}}\left(f-f_{h, \delta} ; x, y\right)\right| \\
& +\rho(x, y)\left|\left(S_{m, n}^{q_{1}, q_{2}} f_{h, \delta}\right)(x, y)-f_{h, \delta}(x, y)\right| \\
& +\rho(x, y)\left|f_{h, \delta}(x, y)-f(x, y)\right| .
\end{aligned}
$$

Inequalities (48) and (53) imply that

$$
\begin{aligned}
& \rho(x, y)\left|S_{m, n}^{q_{1}, q_{2}}\left(f-f_{h, \delta} ; x, y\right)\right| \\
& \leq\left\|S_{m, n}^{q_{1}, q_{2}}\left(f-f_{h, \delta} ; x, y\right)\right\|_{2} \\
& \quad \leq \frac{10}{3}\left\|f-f_{h, \delta}\right\|_{2} \leq \frac{10}{3} \omega_{f}(h, \delta) .
\end{aligned}
$$

Let $C_{2}^{1}\left(\mathbb{R}_{+}^{2}\right)$ be the class of all functions in which partial derivatives belong to $C_{2}\left(\mathbb{R}_{+}^{2}\right)$. Since $f_{h, \delta} \in C_{2}^{1} \mathbb{R}_{+}^{2}$ and $S_{m, n}^{q_{1}, q_{2}}$ given by (10) is linear and monotone, we get

$$
\begin{aligned}
\rho(x, y)\left|\left(S_{m, n}^{q_{1}, q_{2}} f_{h, \delta}\right)(x, y)-f_{h, \delta}(x, y)\right| \\
=\rho(x, y)\left|S_{m, n}^{q_{1}, q_{2}}\left(\int_{x}^{t} \frac{\partial}{\partial u} f_{h, \delta}(u, s) d u ; x, y\right)\right| \\
+\rho(x, y)\left|S_{m, n}^{q_{1}, q_{2}}\left(\int_{y}^{s} \frac{\partial}{\partial v} f_{h, \delta}(x, s) d v ; x, y\right)\right| \\
\leq \rho(x, y) S_{m, n}^{q_{1}, q_{2}}\left(\left|\int_{x}^{t} \frac{\partial}{\partial u} f_{h, \delta}(u, s) d u\right| ; x, y\right) \\
\quad+\rho(x, y) S_{m, n}^{q_{1}, q_{2}}\left(\left|\int_{y}^{s} \frac{\partial}{\partial v} f_{h, \delta}(x, s) d v\right| ; x, y\right) .
\end{aligned}
$$

Then, by definition of norm $\|\cdot\|_{2}$ and the first mean value theorem for integration, we have

$$
\begin{aligned}
\left|\int_{x}^{t} \frac{\partial}{\partial u} f_{h, \delta}(u, s) d u\right| & \leq\left|\int_{x}^{t} \rho(u, s)\right| \frac{\partial}{\partial u} f_{h, \delta}(u, s)\left|\frac{d u}{\rho(u, s)}\right| \\
& \leq\left\|\frac{\partial}{\partial x} f_{h, \delta}\right\|_{2}\left|\int_{x}^{t} \frac{d u}{\rho(u, s)}\right| \\
& =\left\|\frac{\partial}{\partial x} f_{h, \delta}\right\|_{2}|t-x| \frac{1}{\rho(\xi, s)}, \quad \xi \in(x, t) \\
& \leq\left\|\frac{\partial}{\partial x} f_{h, \delta}\right\|_{2}|t-x|\left(\frac{1}{\rho(x, s)}+\frac{1}{\rho(t, s)}\right) .
\end{aligned}
$$

Following the same way, one finds

$$
\begin{aligned}
& \left|\int_{y}^{s} \frac{\partial}{\partial v} f_{h, \delta}(x, s) d v\right| \\
& \quad \leq\left\|\frac{\partial}{\partial y} f_{h, \delta}\right\|_{2}|s-y|\left(\frac{1}{\rho(x, y)}+\frac{1}{\rho(x, s)}\right) .
\end{aligned}
$$


Using inequalities (59), (61), and (60), since $S_{m, n}^{q_{1}, q_{2}}$ is linear and monotone, we get

$$
\begin{aligned}
\rho(x, y)\left|\left(S_{m, n}^{q_{1}, q_{2}} f_{h, \delta}\right)(x, y)-f_{h, \delta}(x, y)\right| \\
\leq \rho(x, y)\left\|\frac{\partial}{\partial x} f_{h, \delta}\right\|_{2} S_{m, n}^{q_{1}, q_{2}}\left(\frac{|t-x|}{\rho(x, s)} ; x, y\right) \\
\quad+\rho(x, y)\left\|\frac{\partial}{\partial x} f_{h, \delta}\right\|_{2} S_{m, n}^{q_{1}, q_{2}}\left(\frac{|t-x|}{\rho(t, s)} ; x, y\right) \\
+\rho(x, y)\left\|\frac{\partial}{\partial y} f_{h, \delta}\right\|_{2} S_{m, n}^{q_{1}, q_{2}}\left(\frac{|s-y|}{\rho(x, y)} ; x, y\right) \\
\quad+\rho(x, y)\left\|\frac{\partial}{\partial y} f_{h, \delta}\right\|_{2} S_{m, n}^{q_{1}, q_{2}}\left(\frac{|s-y|}{\rho(x, s)} ; x, y\right) .
\end{aligned}
$$

From Lemmas 1 and 5 and (36), we can write the following:

$$
\begin{aligned}
\rho(x, y) \mid & \left|\left(S_{m, n}^{q_{1}, q_{2}} f_{h, \delta}\right)(x, y)-f_{h, \delta}(x, y)\right| \\
\leq & \left\|\frac{\partial}{\partial x} f_{h, \delta}\right\| \frac{10}{3} \frac{q_{1}}{[2]_{q_{1}}[m]_{q_{1}}} \\
& +\left\|\frac{\partial}{\partial x} f_{h, \delta}\right\|_{2} \frac{10}{3} \frac{\Gamma\left(x, q_{1}\right)}{[m]_{q_{1}}} \\
& +\left\|\frac{\partial}{\partial y} f_{h, \delta}\right\|_{2} \frac{q_{2}}{[2]_{q_{2}}[n]_{q_{2}}} \\
& +\left\|\frac{\partial}{\partial y} f_{h, \delta}\right\|_{2} \frac{10}{3} \frac{\Gamma\left(y, q_{2}\right)}{[n]_{q_{2}}},
\end{aligned}
$$

where

$$
\Gamma(x, q)=\left(\frac{1}{q}+1+\frac{q}{[2]_{q}}\right) x^{2}+\left(1+\frac{3 q}{[2]_{q}}+\frac{2 q^{2}}{[3]_{q}}\right) x+\frac{q^{3}}{[4]_{q}}
$$

is a polynomial of degree 2 . Then, by inequalities (54), we have

$$
\begin{aligned}
\rho(x, y) \mid & \left(S_{m, n}^{q_{1}, q_{2}} f_{h, \delta}\right)(x, y)-f_{h, \delta}(x, y) \mid \\
\leq & {\left[\frac{2}{h} \frac{10}{3}\left(\frac{q_{1}}{[2]_{q_{1}}[m]_{q_{1}}}+\frac{\Gamma\left(x, q_{1}\right)}{[m]_{q_{1}}}\right)\right.} \\
& \left.+\frac{2}{\delta}\left(\frac{q_{2}}{[2]_{q_{2}}[n]_{q_{2}}}+\frac{10}{3} \frac{\Gamma\left(y, q_{2}\right)}{[n]_{q_{2}}}\right)\right] \omega_{f}(h, \delta) .
\end{aligned}
$$

Finally, we write from (53)

$$
\rho(x, y)\left|f_{h, \delta}(x, y)-f(x, y)\right| \leq\left\|f_{h, \delta}-f\right\|_{2} \leq \omega_{f}(h, \delta) .
$$

If we go back to (57) and take $h=\phi\left(x, q_{1}\right) /[m]_{q_{1}}, \delta=$ $\phi\left(y, q_{2}\right) /[n]_{q_{2}}$, then the proof is completed. that

We replace $q_{1}$ and $q_{2}$ in (10) by sequences $\left(q_{1, m}\right),\left(q_{2, n}\right)$ so

$$
\begin{aligned}
& \lim _{m \rightarrow \infty} q_{1, m}=1, \quad \lim _{n \rightarrow \infty} q_{2, n}=1, \\
& \lim _{m \rightarrow \infty} \frac{1}{[n]_{q_{1, m}}}=0, \quad \lim _{n \rightarrow \infty} \frac{1}{[n]_{q_{2, n}}}=0 .
\end{aligned}
$$

So, $(h, \delta) \rightarrow\left(0^{+}, 0^{+}\right)$as $n, m \rightarrow \infty$. Knowing that modulus of smoothness function $\omega_{f}$ satisfies the property $\lim _{(h, \delta) \rightarrow\left(0^{+}, 0^{+}\right)} \omega_{f}(h, \delta)=0$, from Theorem 6 , we deduce the following result.

Theorem 7. Let $(m, n) \in \mathbb{N} \times \mathbb{N}$ and let $\left(q_{1, m}\right),\left(q_{2, n}\right)$ be sequences in the interval $(0,1)$ satisfying $(67)$. Let the operator $S_{m, n}^{q_{1}, q_{2}}$ given by $(10)$ and $f \in C_{2}\left(\mathbb{R}_{+}^{2}\right) q_{R}$-integrable function. For any $(x, y) \in \mathbb{R}_{+}^{2}$ the pointwise convergence takes place

$$
\lim _{m, n \rightarrow \infty}\left(S_{m, n}^{q_{1, m}, q_{2, n}} f\right)(x, y)=f(x, y)
$$

If $K_{1}, K_{2}$ are compact intervals included in $\mathbb{R}_{+}$, then (68) holds uniformly on the domain $K_{1} \times K_{2}$.

\section{Voronovskaya-Type Theorem}

We will prove the Voronovskaya-type theorem.

Theorem 8. Let $\left(q_{1, n}\right),\left(q_{2, n}\right)$ be sequences in the interval $(0,1)$ satisfying (67). Suppose that $f \in C_{2}^{2}\left(\mathbb{R}_{+}^{2}\right)$ is the class of all functions in which the second partial derivatives belong to $C_{2}\left(\mathbb{R}_{+}^{2}\right)$ and $q_{R}$-integrable function. Then, for every $(x, y) \in$ $\mathbb{R}_{+}^{2}$, one has

$$
\begin{aligned}
\lim _{n \rightarrow \infty} n & \left\{S_{n, n}^{q_{1, n}, q_{2, n}}(f ; x, y)-f(x, y)\right\} \\
= & \frac{x}{2} f_{x x}(x, y)+\frac{y}{2} f_{y y}(x, y) \\
& +\frac{1}{2} f_{x}(x, y)+\frac{1}{2} f_{y}(x, y) .
\end{aligned}
$$

Proof. Let $f \in C_{2}^{2}\left(\mathbb{R}_{+}^{2}\right)$ and $q_{R}$-integrable function and let $\left(x_{0}, y_{0}\right) \in \mathbb{R}_{+}^{2}$ be fixed point. Then, by the Taylor formula, we can write

$$
\begin{aligned}
& f(t, s) \\
& =f\left(x_{0}, y_{0}\right)+f_{x}\left(x_{0}, y_{0}\right)\left(t-x_{0}\right)+f_{y}\left(x_{0}, y_{0}\right)\left(s-y_{0}\right) \\
& +\frac{1}{2}\left\{f_{x x}\left(x_{0}, y_{0}\right)\left(t-x_{0}\right)^{2}+2 f_{x y}\left(x_{0}, y_{0}\right)\right. \\
& \left.\quad \times\left(t-x_{0}\right)\left(s-y_{0}\right)+f_{y y}\left(x_{0}, y_{0}\right)\left(s-y_{0}\right)^{2}\right\} \\
& +\varphi\left(t, s ; x_{0}, y_{0}\right) \sqrt{\left(t-x_{0}\right)^{4}+\left(s-y_{0}\right)^{4}},
\end{aligned}
$$


where $(t, s) \in \mathbb{R}_{+}^{2}, \varphi(t, s)=\varphi\left(t, s ; x_{0}, y_{0}\right)$ belongs to $C_{2}\left(\mathbb{R}_{+}^{2}\right)$, and $\lim _{(t, s) \rightarrow\left(x_{0}, y_{0}\right)} \varphi(t, s)=0$ for $n \in \mathbb{N}$. From the linearity of $S_{n, n}^{q_{1}, q_{2}}$, we have

$$
\begin{aligned}
& S_{n, n}^{q_{1}, q_{2}}\left(f(t, s) ; x_{0}, y_{0}\right) \\
& =f\left(x_{0}, y_{0}\right)+f_{x}\left(x_{0}, y_{0}\right) S_{n, n}^{q_{1}, q_{2}}\left(t-x_{0} ; x_{0}\right) \\
& \quad+f_{y}\left(x_{0}, y_{0}\right) S_{n, n}^{q_{1}, q_{2}}\left(s-y_{0} ; y_{0}\right) \\
& \quad+\frac{1}{2}\left\{f_{x x}\left(x_{0}, y_{0}\right) S_{n, n}^{q_{1}, q_{2}}\left(\left(t-x_{0}\right)^{2} ; x_{0}\right)\right. \\
& \quad+2 f_{x y}\left(x_{0}, y_{0}\right) S_{n, n}^{q_{1}, q_{2}}\left(t-x_{0} ; x_{0}\right) S_{n, n}^{q_{1}, q_{2}}\left(s-y_{0} ; y_{0}\right) \\
& \left.\quad+f_{y y}\left(x_{0}, y_{0}\right) S_{n, n}^{q_{1}, q_{2}}\left(\left(s-y_{0}\right)^{2} ; y_{0}\right)\right\} \\
& \quad+S_{n, n}^{q_{1}, q_{2}}\left(\varphi(t, s) \sqrt{\left(t-x_{0}\right)^{4}+\left(s-y_{0}\right)^{4}} ; x_{0}, y_{0}\right) .
\end{aligned}
$$

By Lemma 1 and since the sequences $q_{1, n}, q_{2, n}$ satisfy (67), we obtain

$$
\begin{gathered}
\lim _{n \rightarrow \infty} n S_{n, n}^{q_{1, n}, q_{2, n}}\left(t-x_{0} ; x_{0}\right)=\frac{1}{2}=\lim _{n \rightarrow \infty} n S_{n, n}^{q_{1, n}, q_{2, n}}\left(s-y_{0} ; y_{0}\right), \\
\lim _{n \rightarrow \infty} n S_{n, n}^{q_{1, n}, q_{2, n}}\left(\left(t-x_{0}\right)^{2} ; x_{0}\right)=x_{0}, \\
\lim _{n \rightarrow \infty} n S_{n, n}^{q_{1, n}, q_{2, n}}\left(\left(s-y_{0}\right)^{2} ; y_{0}\right)=y_{0} .
\end{gathered}
$$

By the Hölder inequality, we have

$$
\begin{aligned}
\left|S_{n, n}^{q_{1}, q_{2}}\left(\varphi(t, s) \sqrt{\left(t-x_{0}\right)^{4}+\left(s-y_{0}\right)^{4}} ; x_{0}, y_{0}\right)\right| \\
\leq\left\{S_{n, n}^{q_{1}, q_{2}}\left(\varphi^{2}(t, s) ; x_{0}, y_{0}\right)\right\}^{1 / 2} \\
\quad \times\left\{S_{n, n}^{q_{1}, q_{2}}\left(\left(t-x_{0}\right)^{4} ; x_{0}\right)+S_{n, n}^{q_{1}, q_{2}}\left(\left(s-y_{0}\right)^{4} ; y_{0}\right)\right\}^{1 / 2} .
\end{aligned}
$$

By properties of $\varphi$ and Theorem 7, we get

$$
\lim _{n \rightarrow \infty} S_{n, n}^{q_{1, n}, q_{2, n}}\left(\varphi^{2}(t, s) ; x_{0}, y_{0}\right)=\varphi^{2}\left(x_{0}, y_{0}\right)=0 \text {. }
$$

From the foregoing facts and using (12) and (38), we obtain

$$
\lim _{n \rightarrow \infty} n S_{n, n}^{q_{1, n}, q_{2, n}}\left(\varphi(t, s) \sqrt{\left(t-x_{0}\right)^{4}+\left(s-y_{0}\right)^{4}} ; x_{0}, y_{0}\right)=0
$$

Then, using (72) and (75), we reproduce from (71)

$$
\begin{aligned}
\lim _{n \rightarrow \infty} n & \left\{S_{n, n}^{q_{1, n}, q_{2, n}}\left(f(t, s) ; x_{0}, y_{0}\right)-f\left(x_{0}, y_{0}\right)\right\} \\
= & \frac{x_{0}}{2} f_{x x}\left(x_{0}, y_{0}\right)+\frac{y_{0}}{2} f_{y y}\left(x_{0}, y_{0}\right) \\
& +\frac{1}{2} f_{x}(x, y)+\frac{1}{2} f_{y}(x, y) .
\end{aligned}
$$

Thus, the proof is completed for $f \in C_{2}^{2}\left(\mathbb{R}_{+}^{2}\right)$.

\section{References}

[1] A. Lupaş, "A q-analogue of the Bernstein operator," in Seminar on Numerical and Statistical Calculus (Cluj-Napoca, 1987), vol. 87 of Preprint, pp. 85-92, University of Cluj-Napoca, ClujNapoca, Romania, 1987.

[2] G. M. Phillips, "Bernstein polynomials based on the $q$-integers," Annals of Numerical Mathematics, vol. 4, no. 1-4, pp. 511-518, 1997.

[3] T. Trif, "Meyer-König and Zeller operators based on the q-integers," Revue d'Analyse Numérique et de Théorie de l'Approximation, vol. 29, no. 2, pp. 221-229, 2000.

[4] O. Doğru and O. Duman, "Statistical approximation of MeyerKönig and Zeller operators based on q-integers," Publicationes Mathematicae Debrecen, vol. 68, no. 1-2, pp. 199-214, 2006.

[5] A. Aral and O. Doğru, "Bleimann, Butzer, and Hahn operators based on the q-integers," Journal of Inequalities and Applications, vol. 2007, Article ID 79410, 12 pages, 2007.

[6] N. I. Mahmudov and P. Sabancigil, " $q$-parametric Bleimann Butzer and Hahn operators," Journal of Inequalities and Applications, vol. 2008, Article ID 816367, 15 pages, 2008.

[7] A. Aral, "A generalization of Szász-Mirakyan operators based on q-integers," Mathematical and Computer Modelling, vol. 47, no. 9-10, pp. 1052-1062, 2008.

[8] N. I. Mahmudov, "Approximation by the q-Szász-Mirakjan operators," Abstract and Applied Analysis, vol. 2012, Article ID 754217, 16 pages, 2012.

[9] N. I. Mahmudov, "On q-parametric Szász-Mirakjan operators," Mediterranean Journal of Mathematics, vol. 7, no. 3, pp. 297-311, 2010.

[10] O. Agratini and C. Radu, "On $q$-Baskakov-Mastroianni operators," The Rocky Mountain Journal of Mathematics, vol. 42, no. 3, pp. 773-790, 2012.

[11] N. I. Mahmudov, "Statistical approximation of Baskakov and Baskakov-Kantorovich operators based on the q-integers," Central European Journal of Mathematics, vol. 8, no. 4, pp. 816-826, 2010.

[12] D. D. Stancu, "A new class of uniform approximating polynomial operators in two and several variables," in Proceedings of the Conference on the Constructive Theory of Functions, pp. 443455, Akadémiai Kiadó, Budapest, Hungary, 1972.

[13] D. Barbosu, "Some generalized bivariate Bernstein operators," Mathematical Notes, vol. 1, no. 1, pp. 3-10, 2000.

[14] O. Doğru and V. Gupta, "Korovkin-type approximation properties of bivariate q-Meyer-König and Zeller operators," Calcolo, vol. 43, no. 1, pp. 51-63, 2006.

[15] O. Agratini, "Bivariate positive operators in polynomial weighted spaces," Abstract and Applied Analysis, vol. 2013, Article ID 850760, 8 pages, 2013.

[16] S. Marinković, P. Rajković, and M. Stanković, "The inequalities for some types of $q$-integrals," Computers \& Mathematics with Applications, vol. 56, no. 10, pp. 2490-2498, 2008.

[17] G. E. Andrews, R. Askey, and R. Roy, Special Functions, vol. 71 of Encyclopedia of Mathematics and its Applications, Cambridge University Press, Cambridge, UK, 1999.

[18] V. Kac and P. Cheung, Quantum Calculus, Universitext, Springer, New York, NY, USA, 2002. 


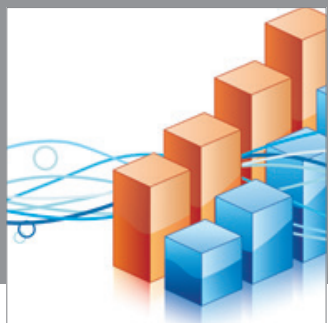

Advances in

Operations Research

mansans

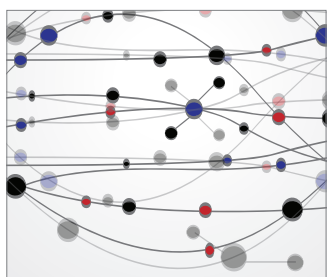

The Scientific World Journal
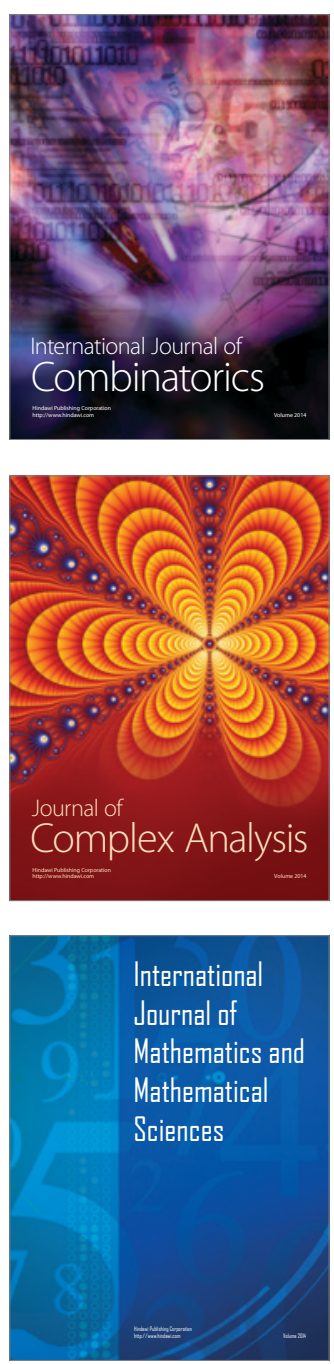
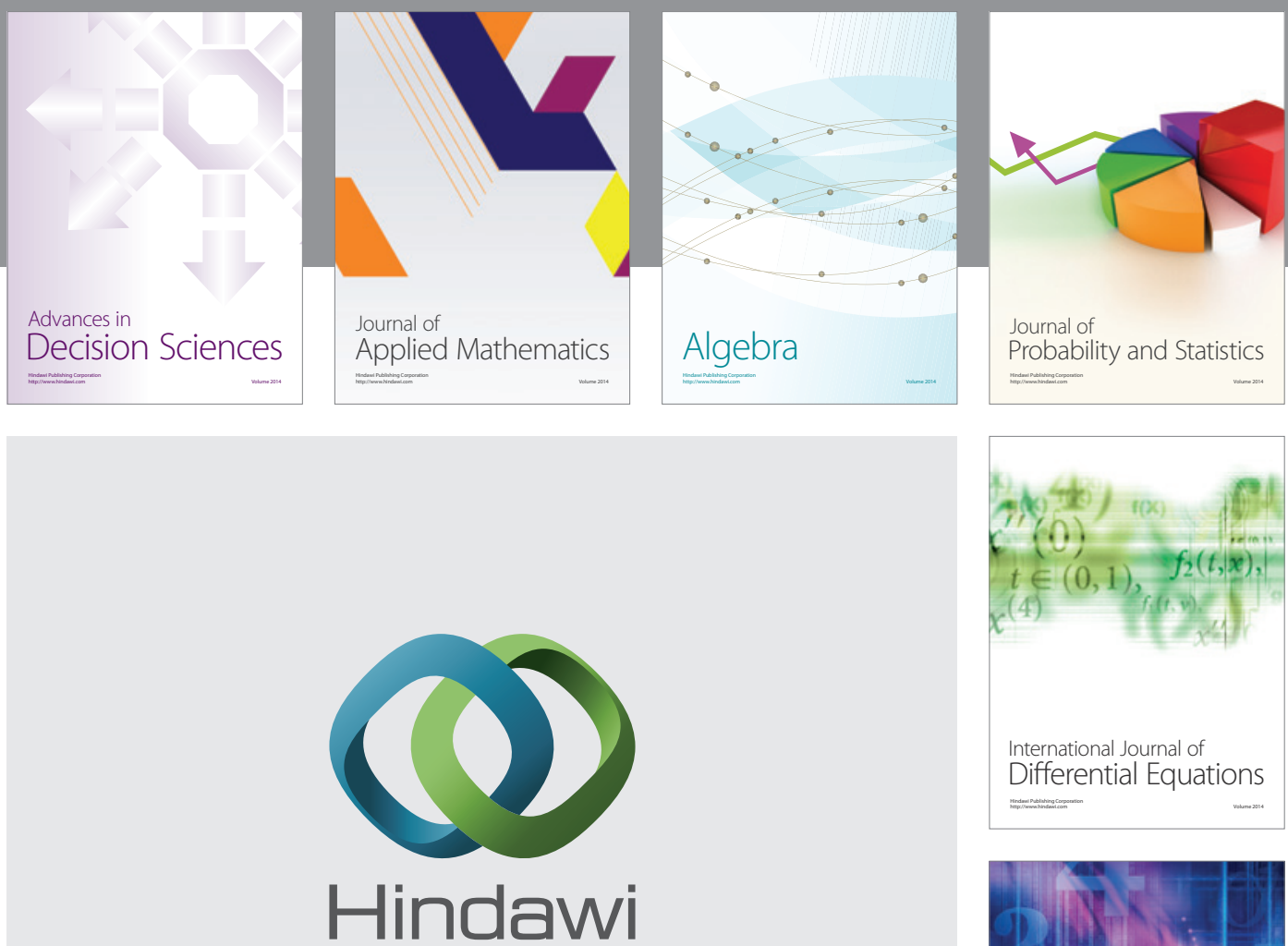

Submit your manuscripts at http://www.hindawi.com
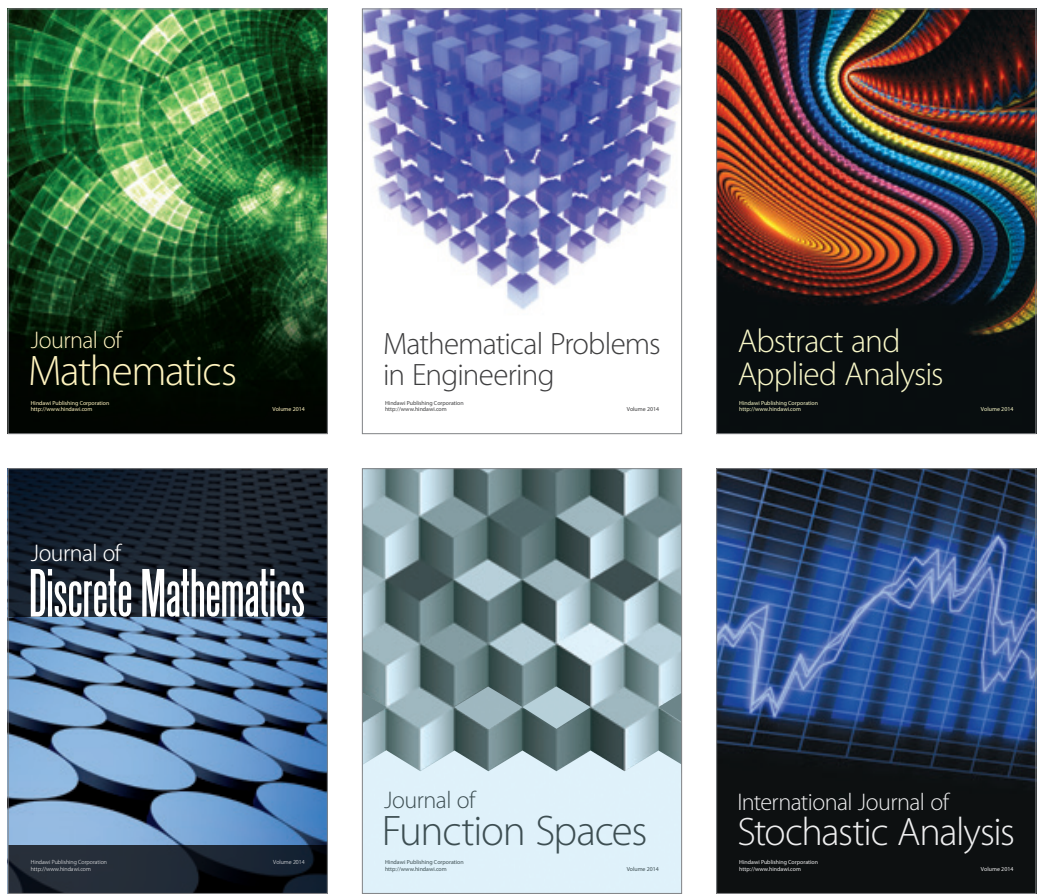

Journal of

Function Spaces

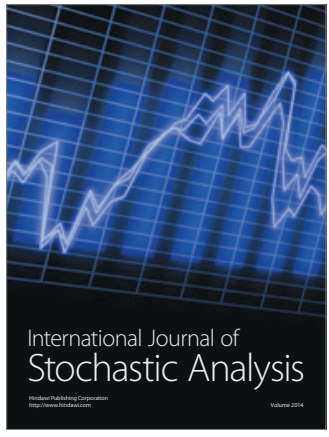

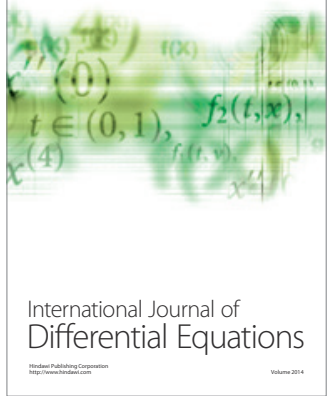
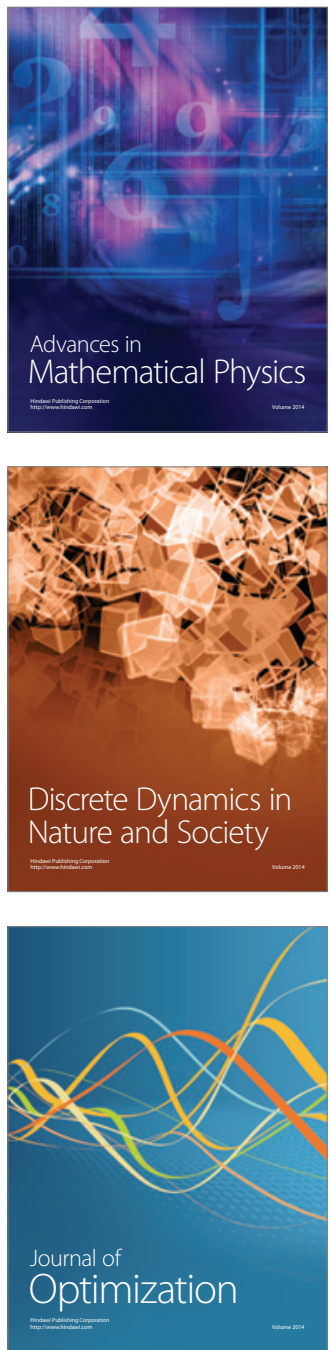